\section{LA INFANCIA EN EL ESQUEMA MARAÑONIANO DE LAS EDADES DE LA VIDA DEL HOMBRE}

\author{
Rosa Ballester \\ Unidad de Historia de la Ciencia \\ Facultad de Medicina \\ Universidad Miguel Hernández \\ rosa.ballester@umh.es
}

\begin{abstract}
Cómo citar este artículo/ Citation: Ballester, R. (2013). La infancia en el esquema marañoniano de las edades de la vida del hombre. Arbor, 189(759):a006. doi: http://dx.doi.org/ 10.3989/ arbor.2013.759n1005
\end{abstract}

Recibido: 3 septiembre 2012; Aceptado: 1 octubre 2012.

RESUMEN: La ingente obra científica de Marañón incluye también una serie de aportaciones sobre el tema de las edades de la vida humana. Desde acercamientos biológicos a los de cariz médico-social, como la eugenesia, el autor presenta su particular visión de la infancia definida de acuerdo a una serie de criterios fundamentados, en gran medida, en su pensamiento endocrinológico. El peso de la tradición de la Antigüedad clásica y de autores renacentistas como el español Huarte de San Juan, está presente como marco de referencia histórico y como una forma de reflexionar sobre situaciones actuales.

PALABRAS CLAVE: Edades de la vida; eugenesia; mortalidad infantil; emociones; infancia.

"Nos explica también [Huarte de San Juan], el distinto temperamento que corresponde a cada edad, el ingenio diverso del ser humano en cada etapa de su ciclo vital. El niño es blando y tierno y por ello, caritativo, liberal, casto y alegre. En la adolescencia, el temperamento se va templando y el ingenio se hace moldeable como la cera: es la edad eminentemente pasiva y, por lo tanto, de responsabilidad máxima para los que tenemos la misión de educar" (Marañón, 1945, 141).

\section{CHILDHOOD IN MARAÑON'S SCHEME OF THE AGES OF LIFE}

Copyright: (C) 2013 CSIC. Este es un artículo de acceso abierto distribuido bajo los términos de la licencia Creative Commons Attribution-Non Commercial (by-nc) Spain 3.0.

ABSTRACT: The huge volume of scientific work by Marañón includes a series of contributions on the subject of the ages of human life. From biological approaches to medical/social issues, such as eugenics, the author presents his individual vision of children defined according to a set of criteria grounded largely in his endocrinological theories. The weight of tradition from classical and Renaissance authors such as the Spaniard Huarte de San Juan, is present both as a historical reference framework and as a means of reflecting on current situations.

KEYWORDS: Ages of life; eugenics; child mortality; emotions; childhood.

La concepción renacentista de las edades de la vida del hombre, presente en la obra de Huarte de San Juan, con sus propias peculiaridades, le sirve a Marañón como leit motiv para hacer, como él mismo indica, "examen actual de un examen antiguo". Y en dicho examen, está haciendo también una lectura en clave de actualidad cuando se refiere, por ejemplo, a los problemas de Huarte con la censura y las ortodoxias estrictas y su reivindicación de un pensamiento libre, no sujeto a ese tipo de ataduras externas, en 
sintonía con su conocido pensamiento antidogmático. (Marañón, 1957, 15-53) y, en general, guarda estrecha relación con su trayectoria biográfica (López Vega, 2010a) y el conjunto de sus aportaciones y publicaciones (López Vega, 2010b). Por otro lado interpreta Marañón este examen de ingenios, no como orientación profesional - la forma habitual de entenderlo- sino, enlazando con sus ideas sobre la vocación, como el estudio fundamental de la constitución de las personas, la razón biológica más íntima de su afición, término que entiende Marañón como amor a la cosa elegida y de ahínco y eficacia en dicho amor.

El asunto de la temporalidad y de las edades de la vida, está presente en la obra marañoniana, bien como marco general, bien como estudio de unos segmentos de edad determinados. Ciertamente, su fundamental estudio de la "edad crítica", el climaterio, es, con diferencia, el periodo de la vida al que dedica un número más amplio de trabajos. No sucede lo mismo con la infancia que no aparece sino tangencialmente. Los motivos son fáciles de entender: su práctica clínica no se consagró precisamente a la pediatría, entre otras cosas, porque en aquellos momentos, la especialidad y los especialistas tenían ya su campo de acción propio y, en gran medida, monopolizaban ya esta parcela del saber y de la práctica médicas (Ballester, 1979). Una constante en la obra de Marañón es la convicción de que la diversidad de la ciencia contemporánea superaría de tal modo la capacidad investigadora del entendimiento humano, que no quedaría espacio suficiente para que una sola persona pueda abarcarlo e informarse suficientemente como para poder realizar críticas a los trabajos científicos de otros colegas (Marañón, 1922, 1-2). Por ello, la división de trabajo y la especialización se transforman en una necesidad inherente al mismo desarrollo científico. Sin embargo, en el tema que nos ocupa, son muchas y muy interesantes las reflexiones que aparecen en la obra marañoniana sobre las primeras etapas de la vida humana, asunto que nos proponemos analizar, no sin antes, trazar brevemente la trayectoria evolutiva de dicho concepto desde su punto de partida al periodo en el que Marañón se ocupa de este particular.

\section{LAS EDADES DE LA VIDA DEL HOMBRE}

La temporalidad es una constante en el pensamiento científico europeo-occidental. Hay dos formas del tiempo que es útil distinguir: el tiempo histórico y el tiempo personal, el tiempo biográfico. Es evidente que el tiempo es continuo pero también lo es que la continuidad no excluye la articulación. El tiempo histórico está articulado en dos sentidos. Por un lado, tiene una articulación menor que son las generaciones, $y$ una articulación mayor, una especie de macroestructura que son las épocas (Marías, 1949). Algo parecido ocurre en la vida: la vida es también continua desde el nacimiento hasta la muerte pero está articulada en edades. Ciertamente, desde las culturas más antiguas se ha reconocido la pluralidad de las edades y se ha tratado de definirlas. En otro lugar nos hemos acercamos a este tema, intentando reconstruir el proceso mediante el cual se planteó, en términos científicomédicos, el tópico de las edades del hombre en algunas de las fuentes médicas españolas de los siglos XV a XVIII (Ballester, 2001).

¿De dónde procede dicho tópico de las edades de la vida del hombre? Sin ningún género de dudas, su punto de partida hay que buscarlo en la Antigüedad Clásica y, como sucede con el resto del conjunto de saberes biológicos y médicos, en el contexto armonizador que fue la teoría humoral. A partir de ahí, va a ser vehiculizado en el largo y complejo proceso de transmisión de la ciencia clásica occidental pasada por el tamiz de la ciencia árabe y del mundo bizantino para desembocar en la medicina escolástica. Al exponer la doctrina de las edades de la vida del hombre tras el estudio de las cosas naturales o fisiológicas, la Isagoge de loannitius, el texto escolar más importante de la historia del galenismo, señala lo siguiente:

"Cuatro son las edades, a saber, adolescencia, juventud, senectud y decrepitud. La adolescencia es de complexión cálida y húmeda, en ella crece y aumenta el cuerpo hasta llegar a los veinticinco o treinta años. A ella le sigue la juventud, que es cálida y seca y que conserva en su perfección el cuerpo sin disminución de sus fuerzas; ésta acaba a los treinta y cinco o cuarenta años. A continuación viene la senectud, fría y seca, en la que el cuerpo empieza a disminuir y decrecer algo, sin que falte, no obstante, la fuerza; dura hasta los cincuenta y cinco o sesenta años. Le sucede la decrepitud, con su concurrencia de humor flemático frío y húmedo; en ella se hace presente la carencia de fuerza y ella, con el transcurso de los años, pone fin a la vida" (Cfr. Gracia y Vidal, 1974-75, 303).

En el texto se dice que la juventud sigue a la adolescencia, que es cálida y seca conservando el cuerpo sin disminución de sus fuerzas. La expresión, tan escueta, no es fácil de entender. ¿Cuál es su significado? Por el contexto, se deduce que hasta los cuarenta años, una vez acabada la juventud, empieza la "disminución", el "decrecimiento" del cuerpo humano, en tanto que en la adolescencia, por el contrario, el cuerpo "aumenta". Parece que el periodo de la juventud - entre los 25-40 años- es "perfecto", porque en él ha finalizado ya el proceso de crecimiento y no se ha iniciado todavía el de disminución o decrecimiento. En términos aristotélicos, la juventud es el periodo de la vida del ser humano que transcurre entre el final del proceso de "generación" y el inicio del de "corrupción". Ahora bien, en la filosofía aristotélica y, por extensión, en toda la filosofía antigua, la "naturaleza" de un ser o una cosa sólo existe perfecta una vez finalizada la generación y 
aún no iniciada la corrupción. Lo cual plantea un grave problema, el de si el niño es un ser natural, es decir, el de qué sentido tiene la expresión "naturaleza" aplicada al niño (Gracia, 1985, 11-23).

De forma magistral, Pedro Laín ha reflexionado sobre la formación del concepto de naturaleza humana (Laín, 1989). Recurriendo a Sócrates indica que dicho concepto no es como usualmente se indica: la naturaleza de una cosa sería la realidad que ésta tiene al nacer; así, por ejemplo, la naturaleza del trigo sería el tallo que nace de la semilla y la naturaleza del hombre, la realidad de éste tras el parto. Hay, empero, otra forma de ver las cosas: que la "generación" es un proceso largo y no concluye hasta que todas las potencialidades han quedado actualizadas. No es un azar que el tratado hipocrático De natura pueri dedique gran parte de sus páginas a establecer una muy minuciosa comparación entre las plantas y el desarrollo del niño. Como en las plantas, la generación no finaliza hasta el logro de la madurez total (Laín, 1970, 16465). Sócrates fue el hombre que aplicó de modo genial estos principios al caso concreto, de la "naturaleza humana". Efectivamente, la generación del hombre no finaliza en el momento del nacimiento sino mucho después, cuando se alcanza la madurez biológica y psicológica entre los veinticinco y los treinta años. Solo entonces el hombre es un "ser natural", es decir, en la plenitud de sus potencias y facultades, tanto corpóreas como anímicas; solo entonces el hombre puede alcanzar su "perfección", la salud y belleza en el cuerpo y la bondad en el alma. Al hombre le pertenecen por "naturaleza" estas propiedades, la bondad, la belleza, la salud. Más aún, en la naturaleza humana hay una correlación perfecta entre cuerpo y alma y solo, en un cuerpo sano y bello, puede existir un alma buena.

Hasta que el hombre alcanza la madurez y su naturaleza logra la perfección, no puede considerarse ni como sano, ni como bello ni como bueno. El niño, no es, en primer lugar, sano, ya que su complexión carece de temperancia. Como consecuencia, tampoco es beIlo. El niño, en fin, no es bueno. Las cualidades del alma se hallan determinadas, en toda la tradición socrática, por la complexión humoral: no puede haber un alma buena en un cuerpo intemperado. Al niño no le pertenece la virtud como nota constitutiva de su naturaleza $y$, mucho menos, a la niña. Poco a poco hay que ayudar al niño y al joven a que desarrollen las potencialidades de su naturaleza, de tal modo que vayan dejando atrás la enfermedad, la fealdad y la maldad de la infancia y acercándose paulatinamente a la salud, la belleza y la bondad, es decir, a la perfección propia de la edad adulta (Gracia, 1985).

El tema de las edades es importante en otro tipo de estudios como los arqueológicos. Por ejemplo, el estudio de las tumbas infantiles en Europa Occidental durante el periodo medieval muestra cómo había un lugar específico reservado a ellos, estableciéndose la hipótesis de la separación entre los bautizados y los no bautizados. Se inhumaban junto a uno de los muros de las iglesias, o en la periferia del cementerio, dependiendo del estatus social. Estos repartos, por otro lado, están hechos con arreglo al criterio de las edades: los recién nacidos (hasta el mes de vida), los niños de 1 mes a seis o siete años, los niños mayores de esa edad y los adolescentes. A partir del momento en el que el individuo cumple el primer mes, ya puede integrarse dentro de la sociedad, adquiriendo un estatus social intermedio entre el del recién nacido y el adulto. Esta integración se produce tras cumplir los seis años, lo que coincide con el momento en el que se le denomina puer y ya no infans.

A este respecto, los trabajos de Shulamith Shahar (1992), constituyen una importante base de reflexión. Para esta autora, las edades de la vida que aparecen en las obras médicas, literarias y didácticas antiguas y tradicionales, se corresponden con la formación de la personalidad del niño estudiada por Jean Piaget. Los datos arqueológicos confirman que la transición de infans a puer, como acabamos de comentar, se produce entre los 6 y los siete años. Por ejemplo en textos normativos o penitenciales, aparece esta distinción. Los primeros, por ejemplo, ya deben hacer penitencia de sus pecados, adecuada a su edad, y no los segundos (Garnotel y Fabre, 1997).

En el periodo moderno, Martín Martínez, uno de los médicos españoles más prestigiosos, en su Medicina Escéptica (1748), pone en boca de los personajes ficticios que polemizan en la conversación sexta de la obra, relativa a "que sean las edades y cuantas", el hipocrático, el galénico y el químico un interesante resumen de las ideas que sobre este lugar común, tienen cada uno de ellos. Así el hipocrático, desde una perspectiva poco dogmática, dice las siguientes palabras:

“No gastéis tiempo en eso (en ver el no de edades) pues según los fines que se han propuesto los autores, han dividido tan variadamente las edades, que apenas hay número que no tenga su protección: tal vez, edad se toma por aetas y esto es todo el curso de la vida; tal vez por los dos extremos: mozos y viejos; tal vez por los tres estados de Aristóteles; tal por quatro como Hipócrates, tal por cinco como Platón; otros dan seis edades, otros ocho partiendo partiendo la vejez en vejez y decrepitud y la menor edad en infancia y puericia y también es de Hipócrates esta división. Los astrólogos dan siete por la fingida correspondencia a los planetas, ay quien de siete en siete años admite una edad y hasta doce edades no faltan patronos..." (Martínez, 1748, 69).

Por el contrario, el galénico ofrece el entramado doctrinal de la tradición clásica y, finalmente, el chí- 
mico, ofrece hasta siete edades distintas (infancia, puericia, pubertad, adolescencia, juventud, virilidad y senectud. Las diferencias entre una y otra se realiza con un criterio procedente de la pura observación de los cambios corporales con escasa interpretación doctrinal.

La ruptura definitiva con las tesis tradicionales, en el caso del tópico que nos ocupa, hunde sus raíces en las monografías sobre enfermedades de los niños de finales del siglo XVIII y se consolida dentro del movimiento que, desde los hospitales del París postrevolucionario de inicios del siglo XIX, se extenderá rápidamente a toda Europa y al resto del mundo occidental y que dará lugar, entre otras cosas, a la aparición de la especialidad pediátrica. El estudio del cuerpo infantil en estado de salud y, sobre todo, de enfermedad, a través de la observación clínica sistemática, la búsqueda de signos ciertos para el diagnóstico y la información proporcionada por las autopsias y más tarde, por la medicina de laboratorio, cristalizarán en una nueva visión médica del organismo en estas etapas iniciales de la vida humana. Los niños no son de naturaleza cualitativamente diferente a la de los adultos y las edades se categorizan, desde ese momento, con criterios estrictamente médicos, acordes con la anatomía, la fisiología y los modos peculiares de enfermar característicos de cada fase: recién nacido, lactante, primera y segunda infancia y adolescencia. Una de las claves fundamentales en este proceso, es la correspondencia entre el desarrollo del sistema nervioso que, en todos sus componentes, va adquiriendo una complejidad creciente, y las edades de la vida.

Para cerrar este recorrido histórico y analizar cómo éste tópico de las edades de la vida se expresa en la obra de Marañón, parece oportuno partir de los puntos de vista, encuadrados dentro de lo que se denomina orientación biopatológica, del coordinador y artífice del primer gran tratado de Pediatría (Pfaundler y Schlossmann, 1906), el alemán Meinhard von Pfaundler. Para este autor, es necesario plantearse en estos segmentos de edad, la correlación existente entre las diversas partes del cuerpo, una coordinación que está determinada por unos sustratos de tipo nervioso y endocrinológico perfectamente demostrables por la ciencia contemporánea. Pues bien, toda esta tradición pesará y será uno de los puntos de partida del enfoque que Marañón expresa en sus trabajos relativos a este tema.

\section{LA VARIABILIDAD DE UN CONCEPTO DESDE LA EXPERIEN- CIA CLÍNICA. LA IDENTIDAD DE LA INFANCIA DESDE LA BIOLOGÍA}

No cabe duda del buen conocimiento, por parte de Marañón, de los entresijos conceptuales de la tradición occidental del tópico "edades de la vida del hom- bre" y de sus principales mentores, pero para nuestro autor, y ese matiz es muy importante, en su visión de las edades de la vida, prima el clínico observador y experimentado cuando indica que: "al hablar de las distintas edades de la vida, no seguiremos ninguna de las divisiones de ésta en edades, propuestas por los autores. Todas ellas, fundadas sobre cánones numéricos, nos parecen artificiosas, como lo es todo intento de ajustar a patrones matemáticos, la infinita variabilidad de los fenómenos biológicos [...]. Nosotros, por lo tanto, consideraremos la niñez, el periodo puberal, la juventud, la madurez, el climaterio, la vejez y la senectud sin atenernos a fechas (no tan precisas como las de los Padres de la Iglesia que dividían la vida por múltiplos de seis), pero, ni siquiera, aproximada, ya que cada organismo crece, madura y declina, con individual y características cronología" (Marañón, 1921, 42-43).

La versión marañoniana del esquema de las edades está directamente ligado a lo que fueron, en torno a los años 20 y 30 del pasado siglo, las investigaciones sobre las transformaciones biológicas que tienen lugar en las etapas subsiguientes de la vida extrauterina, en definitiva, sobre el crecimiento humano. El tema es tan importante que nuestro autor le dedica un trabajo monográfico (Marañón, 1953) y otros trabajos que, en su conjunto, han sido analizados recientemente (Ariznavarreta y Fernández Tresguerres, 2003). Dichos estudios sobre la infancia supusieron, en contraste con la tradición heredada, un cambio cualitativo en el sentido de que el niño dejó de verse como un adulto en miniatura, para pasar a ser contemplado desde su propia identidad, como un organismo vivo cuya peculiaridad residía en el conjunto de mecanismos fisiológicos que intervenían en aquello que era propio de esas edades, el crecimiento (Tanner, 1981). La indagación sobre la fisiología del crecimiento proporcionó argumentos sólidos para marcar el tránsito de una a otra de las edades, para valorar el estado de gravedad de un caso clínico determinado y como elemento diferenciador entre enfermedades agudas y crónicas. El conocimiento en profundidad del cuerpo de los niños permitía las comparaciones entre ellos $-\mathrm{y}$ no con los adultos - y planteaba la posibilidad de establecer el arquetipo del niño saludable como patrón de referencia con el que podían ser comparados, para ver el grado de adecuación del niño o niños que se estuvieran estudiando, a este arquetipo ideal (Ballester y Perdiguero, 2003).

Con la sólida base proporcionada por los estudios experimentales realizados sobre larvas de ranas, y que Marañón considera podían ser aplicados a los organismos superiores, y con la concurrencia de otro tipo de factores exógenos como el clima o la alimentación, nuestro médico elabora sus propias conclusiones. Lo fundamental de las mismas se resume en lo 
siguiente: el crecimiento y desarrollo infantiles se deben a los cambios metabólicos y fisiológicos generales que en el organismo se producen por la acción de las hormonas. Las diferentes glándulas de secreción interna ejercen un papel decisivo sobre el desarrollo del esqueleto y la morfología general extrauterina. La hipótesis expuesta permite además explicar hechos bien conocidos con anterioridad al siglo $X X$ y es que cuando el tiroides enferma en las primeras edades de la vida o cuando se extirpa esa glándula a un animal joven, uno de los efectos más constante y marcados es la "inhibición del crecimiento de los huesos, que quedan estacionados, cortos, sin soldarse los cartílagos de conjunción y con una estructura rara y quebradiza" (Marañón, 1922, 52).

Ahora bien, el sistema endocrino no ejercería su función de manera regular, dentro de límites bien definidos como le sucedería al sistema circulatorio o al aparato locomotor, una vez que la persona ha traspuesto la primera infancia. Por el contrario, del mismo modo que sucede con el sistema nervioso, aunque en mayor intensidad, la actividad de las glándulas endocrinas se desarrollaría a través de oscilaciones en cada fase de la evolución del ser y experimentaría en ciertas épocas, crisis complicadas. De estas oscilaciones del funcionamiento endocrino, dependerían directamente las oscilaciones del crecimiento del esqueleto y de la totalidad del organismo, las diferentes fases de la actividad sexual y las del metabolismo orgánico (Marañón, 1921, 35-56).

Las distintas etapas se pueden individualizar y separar por lo que denomina "actividad funcional del sistema endocrino". En la niñez, hasta los nueve años, cuando empieza a prepararse el organismo del niño para la crisis puberal, la actividad endocrina estaría casi exclusivamente limitada al tiroides y al timo, que regulan el crecimiento esquelético. La glándula genital, la hipófisis y las suprarrenales tendrían en esta época una función muy escasa todavía. La llegada de la pubertad coincidiría con la regresión funcional y anatómica del timo. La glándula hipofisaria entraría entonces en juego y de su actividad dependería el patrón de crecimiento brusco del niño en esos años en los que el incremento de la talla adquiere un ritmo acelerado e irregular (Marañón, 1953).

Desde el punto de vista patológico, Marañón acuña el concepto de "cronopatías" como alteraciones de la cronología normal en el proceso de crecimiento. De forma muy detallada separa lo que son las patologías reales como algunos tipos de infantilismo causados, por ejemplo, los de origen hipofisario o tiroideo, de las evoluciones disarmónicas del crecimiento, las cronopatías propiamente dichas. El tema es tratado en varias entradas diferentes de esta suerte de diccionario médico que es su monumental Manual de diagnóstico etiológico (Marañón, 1961, p. 550-552;
580-582), como cronopatías del desarrollo infantil o cronopatías de la talla, pero siempre desde el enfoque arriba indicado.

\section{LA EDAD Y LAS EMOCIONES}

Las aportaciones de Marañón al tema de la emoción, han sido objeto de estudio monográfico en diferentes ocasiones, como el ciclo de conferencias que tuvo lugar en el marco de la Semana Marañón celebrada en Madrid en 1999 y en estudios consagrados a esta cuestión (Fernández de Molina, 2003).

También en el sistema neuroendocrino, habría que ubicar las emociones, por el paralelismo entre la actividad endocrina y la sensibilidad emocional a lo largo de la vida. De hecho, la evolución de las funciones cerebrales en las primeras edades de la vida y el análisis del desarrollo de la afectividad y, en general, de las emociones, en cada momento de la vida, ocupa un lugar importante en la obra de nuestro autor. Las oscilaciones y características de la emotividad en la edad infantil dependerían fundamentalmente de la situación funcional del sistema endocrinovegetativo, aunque el medio ambiente externo también podría influir. Critica abiertamente la muy difundida y reeditada obra El alma del niño publicada en 1882 (Preyer, 1882), ya que, pese a la formación como fisiólogo de su autor, su darwinismo militante y, sobre todo, su papel como uno de los pioneros de la psicología del desarrollo, considera que tiene escasa relación con la experiencia directa y con las bases biológicas de la emotividad infantil y que más que "el alma del niño" le da la impresión de que Preyer estudia la historia del alma "de un niño", de un solo niño (su propio hijo). Y aquí, una vez más, habla el clínico Marañón y expresa su escepticismo por un rígido esquematismo de las edades. Para nuestro autor, basándose según sus propias palabras, en la experiencia propia, las emociones comenzarían en cada niño a una edad variable y también, por orden variable, siendo artificioso, por consiguiente, todo intento de fijar de forma estricta la cronología de los estados afectivos (Marañón, 1966, 45).

Las emociones instintivas, las ligadas directamente a los instintos primarios como el amor, la cólera, el dolor, la alegría o el miedo, son aquellas que primero aparecen en la evolución y son las que experimentan los niños, en armonía, prosigue Marañón, con la simplicidad de su sistema nervioso y la escasa complicación de su aparato endocrino. En la expresión corporal de dichas emociones, hay un fuerte componente motor, con movimientos de los miembros o de todo el cuerpo semejantes a los grandes reflejos motores y ello es así porque el reflejo es, precisamente, el modo de expresarse la reacción instintiva; de ese modo, el recién nacido en el que solo hay instintos, no es más 
que un puro reflejo (Marañón 1966, 47). El niño expresa su alegría por vivos movimientos de brazos, por saltos y gritos; y la tristeza, por actitudes aparatosas como la de tirarse al suelo; su rostro, en cambio, refleja poco los elementos emocionales, también como sucede en la escala filogenética. En definitiva, emociones escasas, ligadas a los instintos fundamentales, pasajeras y expresadas por grandes actitudes motoras, serían, para Marañón, las características de la emotividad en los primeros años de la vida.

Las oscilaciones de la vida afectiva, desde la niñez a la ancianidad en el esquema marañoniano, se corresponden con las oscilaciones funcionales del sistema neuroendocrino, órgano de la emoción por antonomasia. Cada edad se diferenciaría de las otras por una "fisionomía afectiva" particular, más todavía que por los caracteres anatómicos o por elementos intelectuales. Y el hombre más perfecto, sería aquel en el que estuvieran más marcadas las características de las diferentes etapas porque de la capacidad afectiva, "depende el provecho moral que cada uno recoja de la vida y el rastro útil que deje de su paso por ella" (Marañón, 1966, 55).

\section{EL DEBER DE LAS EDADES}

Bajo este rótulo, surge el Marañón cercano a la psicología, la sociología y la ética. Cada edad tiene su deber, y hay unos deberes "naturales" y otros creados por "artificio humano", o, como él mismo indica, deberes naturales y deberes sociales, respectivamente. Acompasar los dos niveles - el natural y el culturalno le parece a nuestro autor tarea fácil aunque sí sería deseable reajustar la civilización a la naturaleza, como intentaron los mentores de la Encyclopédie francesa y el ilustrado Rousseau, entre otros. La corriente eugenésica contemporánea sería la heredera de este enfoque.

La edad es una preocupación ligada a dos de los instintos fundamentales de la especie humana: el instinto sexual y el instinto del temor a la muerte $y$, sobre todo, es uno de los ejes de la personalidad en cada momento de la vida, puesto que los músculos, el corazón o el sistema nervioso son distintos en cada etapa. Para nuestro autor, también hay deberes ligados a cada momento evolutivo (Marañón, 1931). A este respecto, los deberes de la niñez son meramente pasivos y se reducen a obedecer, porque su condición cronológica le obliga a ello. De hecho, antes de la pubertad, las rebeldías de los niños, que son explosiones transitorias necesarias para la expansión del carácter futuro, tienen un carácter meramente episódico a veces aparatoso, pero se trata de una fuerza que se encarrilla fácilmente a su cauce a través de la persuasión y la paciencia, nunca de la violencia.

\section{MORTALIDAD INFANTIL Y EUGENESIA}

El tema de la difusión de las ideas eugenésicas en España ha sido bien estudiado entre nosotros tanto desde una perspectiva general (Álvarez Peláez, 1995), como específicamente referido a Marañón (Ferrándiz y Lafuente, 1999). En este último trabajo, puede encontrarse, además, un detallado y acertado análisis de todos los escritos del autor referidos a este tema, por lo que solo nos referiremos al mismo de forma sucinta por ser necesaria su inclusión en el marco del tema objeto de nuestro estudio.

Dos son, al menos, los rasgos significativos que confieren una personalidad propia a la medicina occidental de las primeras décadas del siglo XX: la preocupación por la mortalidad infantil que desembocará en las cruzadas o luchas sanitarias en este terreno (Rodríguez y Molero, 1993) y, directamente ligado a este problema, la eugenesia. Los médicos españoles en particular, se sienten legitimados para cumplir un deber patriótico en la lucha contra la mortalidad infantil y la degeneración de la raza (Martínez Vargas, 1908). Dicha perspectiva ocupa un lugar destacado en el tema de la infancia, porque nuestro autor la liga directamente a la lucha contra la mortalidad infantil que habría que combatirla en su raíz: "en la lucha por evitar del número excesivo de hijos", argumento que apuntala con estadísticas propias sobre familias proletarias, cuya publicación provocó, en 1926, según el propio autor, un gran debate social y Marañón fue tachado de inmoral y disolvente. Las cifras eran, para Marañón, absolutamente concluyentes. "En las clases populares de España, entre los obreros y las capas más bajas de la pequeña burguesía, la mortalidad infantil pudiéramos decir que es aceptable — si la muerte de un niño no nos causase siempre una reacción de rebeldía- en tanto que el matrimonio no pasa de los cinco hijos. A partir de ese número, la mortalidad empieza a crecer en proporciones atroces. Ya las familias de nueve hijos dan una mortalidad media que se acerca al sesenta por cien y, por encima de ese número, la muerte se ensaña de forma progresiva en los hogares, a medida que son más numerosos; terminando por llegar al noventa por cien en el tipo medio de esos matrimonios ejemplares y heroicos, aún frecuentes en nuestras clases bajas, en las que la mujer ha concebido, alumbrado y a veces criado más de 17, 18 o 20 criaturas" (Marañón, 1933, 235). En esta lucha, dice, se siente acompañado no solo por las gentes juveniles y progresistas, sino por doctos espíritus de la llamada derecha, imbuidos de sentido conservador, algunos católicos militantes, incluso sacerdotes.

La solución a este problema pasa por seguir las normas marcadas por la eugenesia, "la ciencia de nuestro tiempo y la de más fecundo porvenir" (Marañón, $1928,132)$, a la que defiende en nombre del saber y 
del progreso. Se trataría de educar a las personas en la gran responsabilidad que supone traer hijos al mundo y afrontar, desde los poderes públicos, dos cuestiones: la primera, la necesidad de evitar la procreación en padres que tengan enfermedades transmisibles ("el enfermo debe curarse antes de ser padre, y si no se puede curar, no debe ser padre nunca") (p. 238). En segundo lugar, deben evitarse los premios a las familias muy numerosas: "aquellas que vemos en los retratos de los periódicos como pequeños batallones prestos a morir por la ambición de cualquiera... sino para que se tengan los hijos precisos para poder ser atendidos y no se pierda su esfuerzo en esa herodiada que arrasa cada año a los niños españoles" (p. 238).
A modo de conclusión señalaríamos que la incardinación del tema de las edades de la vida humana y del conocimiento de la primera de las etapas de la misma, la infancia, en la obra de Marañón, hay que entenderla como una aproximación que conjuga diferentes elementos: por un lado, la conciencia de que es necesario conocer el recorrido histórico de los conceptos con la intención de recrearlos y darles un sentido, el propio del contexto en el que se desarrollaron. En segundo lugar, ofrecer una visión actualizada que, basada en la ciencia contemporánea y la medicina de laboratorio, pero siempre bajo el principio rector de la experiencia clínica, fundamente, desde los esquemas de la doctrina de las secreciones internas, la identidad fisiológica y patológica del organismo infantil.

\section{BIBLIOGRAFÍA}

Álvarez Peláez, R (1995). Penetración y difusión de la eugenesia en España. En E. Arquiola y J. Martínez Pérez, Ciencia en expansión. Estudio sobre la difusión de las ideas científicas y médicas en España. Madrid: Editorial Complutense, p.211-232.

Arriznavarreta Ruiz, C. y Fernández Tresguerres, J.A. (2003). El crecimiento y su estudio en la obra de Marañón. En Revisión de la obra médica de Gregorio Marañón. Madrid: Fundación Marañón, p. $161-210$

Balaguer, E. y Ballester, R. (2005). La infancia como valor y como problema en las luchas sanitarias de principios de siglo en España. Dynamis 15: 177-192.

Ballester, R. (1979). La historia clínica pediátrica durante el siglo XIX. Zaragoza: Universidad de Zaragoza.

Ballester, R. (2001). Edades de las mujeres/ edades de la vida del hombre. Tópicos y lugares comunes en la ciencia antigua y tradicional. En P. Pérez Cantó y $\mathrm{M}$. Ortega López, Edades de las mujeres. Madrid: EAIHM/ UAM, p.3-20.

Ballester, R. y Perdiguero, E. (2003). Ciencia e ideología en los estudios sobre crecimiento humano en España y en Francia y en España (1900-1950). Dynamis 23: 61-84.

Fernández de Molina, A. (2003). Contribución de Marañón al tema de la emoción. En VVAA. Revisión de la obra médica de Marañón (ROMM).Ciudad Real: Fundación Gregorio Marañón/ Ediciones Puertollano, p. 349-369.
Ferrándiz, A. y Lafuente, E. (1999). El pensamiento eugénico de Marañón. En R. Álvarez Peláez, Dossier: Estudios sobre eugenesia. Asclepio, 51(2): 133-148.

Garnotel, A. y Fabre V. (1997). La place de l'enfant médiéval dans l'espace des morts. Aport des fouilles du Lunelloi. En Buchet Luc (dir), L'enfant, son corps, son histoire. Sophia Antipolis: APDCA, 1997, p. 9-24

Gracia Guillén, D. y Vidal, J.L. (1974-75). La Isagoge de Ioannitius. Asclepio, 26-27: 267-310.

Gracia Guillén, D. (1985). ¿Es el niño un ser enfermo? Jano, 662: 11-23.

Laín Entralgo, P. (1970). El curso de la vida humana. En La Medicina Hipocrática. Madrid: Revista de Occidente, p. 164165

Laín Entralgo, P. (1989). El cuerpo humano. Estructura actual. Madrid: Espasa Calpe.

López Vega, A. (2010 a). Biografía de Gregorio Marañón. Madrid: Taurus.

López Vega, A. (2010 b). Biobibliografía de Gregorio Marañón. Madrid: Dykinson,

Marañón, G. (1922). Problemas actuales de la doctrina de las secreciones internas. Madrid: Ruiz Hnos. ed.

Marañón, G. (1953). El crecimiento y sus trastornos. Madrid: Espasa Calpe.
Marañón G. (1957). Crítica de la medicina dogmática. En La medicina y nuestro tiempo. Madrid: Espasa Calpe, p. 550552; 580-582.

Marañón, G. (1960). El deber de las edades. En G. Marañón, Ensayos liberales. Madrid: Espasa-Calpe, Colección Austral, p. 71-114

Marañón, G. (1961). Manual de diagnóstico etiológico.11a ed., Madrid: EspasaCalpe.

Marañón, G. (1933). Relación de la eugenesia con la mortalidad infantil. En Obras completas, vol. III, Madrid, Espasa Calpe, 1966, p. 235-239.

Marañón, G. (1966). Sobre la emoción. En Obras completas. vol. III, 1966, p.35-56.

Marías, J. (1949). El método histórico de las generaciones. Madrid: Revista de Occidente.

Martínez, M. (1748). Medicina Sceptica y Cirugía moderna con un tratado de operaciones quirúrgicas. Madrid.

Martínez Vargas, A. (1908). Deberes sociales del médico contemporáneo. $L a$ medicina de los niños, 8, p. 42-46.

Pfaundler, M. von y Schlossman, A. (1906). Handbuch der Kinderkrankheiten. Leipzig: Vogel.

Preyer, W. (1882). Die Seele des Kindes: Beobachtungen über die geistige Entwicklung des Menschen in den 
ersten Lebensjahren. Grieben, Leipzig 1882 (traducción española con un prólogo de D. Martín Navarro). Madrid: Daniel Jorro, 1902.

Rodríguez Ocaña, E. y Molero Mesa, J. (1993). La cruzada por la salud. Las campañas sanitarias del primer tercio del siglo XX en la construcción de una cultura de la salud. En L. Montiel (ed.), La salud en el estado del bienestar. Análisis histórico. Madrid: Editorial Complutense, p.133-148.
Sahar, S. (1992). Childhood in the Middle Ages. London: Routledge, 1992.

Tanner, J.M. (1981). A history of the study of human growth. Cambridge: Cambridge University Press. 